\title{
LE DISPOSITIF DU DRONE
}

\section{Anne-Katrin Weber}

BSN Press | «A contrario »

2019/2 n² 29 | pages 3 à 24

ISSN 1660-7880

ISBN 9782940648115

Article disponible en ligne à l'adresse :

https://www.cairn.info/revue-a-contrario-2019-2-page-3.htm

Distribution électronique Cairn.info pour BSN Press.

(C) BSN Press. Tous droits réservés pour tous pays.

La reproduction ou représentation de cet article, notamment par photocopie, n'est autorisée que dans les limites des conditions générales d'utilisation du site ou, le cas échéant, des conditions générales de la licence souscrite par votre établissement. Toute autre reproduction ou représentation, en tout ou partie, sous quelque forme et de quelque manière que ce soit, est interdite sauf accord préalable et écrit de l'éditeur, en dehors des cas prévus par la législation en vigueur en France. Il est précisé que son stockage dans une base de données est également interdit. 


\title{
Le dispositif du drone
}

\author{
ANNE-KATRIN WEBER
}

$L$ es drones sont partout. Ils s'étalent sur les présentoirs des magasins de jouets et dans les catalogues destinés aux photographes professionnel-le·s; ils intègrent l'équipement des organisations humanitaires et promettent de révolutionner la logistique des transports et des livraisons; ils équipent les forces de police et les armées tout autour du monde. La presse et la publicité les vantent comme la dernière des «nouveautés ${ }^{1}$ (Kaplan 2017). Ce drone-o-rama (ibid.) que nous livrent quasi quotidiennement les enthousiastes de la technologie ne se limite aucunement aux États-Unis, un des leaders du marché (avec Israël et la Chine). La Suisse s'est fait un nom dans le monde des drones civils en adoptant une régulation particulièrement libérale. La livraison quotidienne d'échantillons médicaux entre hôpitaux et laboratoires par drones ${ }^{2}$ est effectivement permise depuis plus d'une année sur le territoire, tandis que la Confédération a récemment autorisé l'utilisation des drones dans l'agriculture, pour l'épandage de pesticides, ce qui fait de la Suisse «le premier pays d'Europe à autoriser les drones pulvérisateurs " ${ }^{3}$. Le Canton de Vaud, qui abrite l'École polytechnique fédérale de Lausanne ainsi que de nombreuses start-up émanant de cette institution, se présente quant à lui comme "drone valley». Il soutient le développement de technologies droniques via la mise à disposition d'infrastructures "adaptées et connectées", une proximité avec les milieux de la recherche universitaire, et des "mesures fiscales et financières axées sur le long-terme " ${ }^{4}$. Cet engouement pour le drone du côté des industries, de la recherche appliquée et des politiques cantonales et fédérales fait largement écho au débat public, où le drone apparaît comme le signe d'un progrès aussi naturel qu'inévitable. Faisant converger les derniers outils numériques (caméras HD, capteurs divers, aviation

\footnotetext{
1 Sauf mention contraire, les traductions sont de l'auteure.

2 https://www.post.ch/fr/notre-profil/innovation/innovations-en-cours/drones, consultéle $1^{\text {er }}$ octobre 2019.

3 https://www.admin.ch/gov/fr/accueil/documentation/communiques.msg-id-75881.html, consulté le $1^{\mathrm{er}}$ octobre 2019.

4 https://www.vaud-economie.ch/Portals/4/Files/Factsheets/FR/Domaines/FR_Drones.pdf, consulté le $1^{\mathrm{er}}$ octobre 2019.
} 
automatisée...) avec l'intelligence artificielle et le machine learning, le drone incarne ainsi aisément la « révolution numérique» (voir Jeannin dans ce numéro).

Parallèlement à cet enthousiasme public, le monde académique s'est rapidement emparé du sujet, en particulier dans l'espace anglo-saxon où les études portant sur la guerre des drones contemporaine foisonnent depuis plusieurs années. À l'image de la publication de Life in the Age of Drone Warfare (Parks \& Kaplan 2017a), ces travaux proposent de repenser la politique du drone au-delà de la discussion de son rôle stratégique pour les pouvoirs militaires à travers une approche critique et interdisciplinaire. Ces

4 recherches mêlent ainsi volontiers géographie politique, relations internationales, sciences politiques et les Sciences and Technology Studies avec des approches féministes et postcoloniales, entre autres. Elles réfléchissent aux prémisses impérialistes qui structurent la guerre des drones, questionnent ses régimes de pouvoir, interrogent l'éthique des assassinats ciblés ou replacent les visibilités spécifiques que produit le drone dans le contexte plus large des images aériennes. Dans l'espace francophone, la publication de Grégoire Chamayou, Théorie du drone, parue en 2013 aux éditions La Fabrique, constitue une référence incontournable pour une analyse du drone armé et des modalités des guerres droniques dans une perspective philosophico-politique.

Cette introduction au numéro 29 d'a contrario souhaite présenter quelques-unes de ces approches afin de cerner les différentes caractéristiques qui définissent le drone et ses utilisations. Sans prétendre à une quelconque exhaustivité, elle vise à en pointer les enjeux éthiques et politiques, mais également à décortiquer le fonctionnement de la violence propre à l'usage des drones dans les conflits contemporains 5 . Dans cette perspective, cette introduction se concentre sur les usages des drones armés américains, largement étudiés par une littérature pluridisciplinaire. Elle suit ainsi le fil des débats académiques émanant d'un pays qui a recours massivement aux drones, et se focalise sur l'un de ses emplois spécifiques, celui dont l'analyse est actuellement la plus développée.

\section{Quelques dates et chiffres}

L'histoire des Unmanned Aerial Vehicles (UAV) remonte au début du siècle passé et aux premiers tests d'avion autopilotés (Zubeldia 2012; Hall Kindervater 2016; voir Weber dans

5 Plusieurs auteur.e.s proposent des discussions stimulantes de la bibliographie existante. Je renvoie en particulier à l'article de Klauser et Pedrozo (2015) qui offre un bilan détaillé et soulève de nouvelles questions de recherche. Pour les personnes intéressées par la littérature francophone, la bibliographie de cette introduction offre quelques piste; je signale en particulier le numéro « Drones, cartographie et images automatisées» de la revue Décadrages. Cinéma, à travers champs (voir Gunti 2014). 
ce numéro). Ce n'est cependant qu'à partir de la guerre du Vietnam que les États-Unis emploient des UAV dans le cadre d'opérations de surveillance. Durant les années 1980, l'armée israélienne intègre les drones dans leur arsenal répressif. Une décennie plus tard, autant Israël que les États-Unis utilisent les drones pour de nombreuses actions militaires, dont l'assassinat ciblé. La "guerre contre le terrorisme» lancée par les ÉtatsUnis à la suite des attentats du 11 septembre 2001 a intensifié l'usage des drones armés. Des drones sont utilisés dès le début octobre en Afghanistan pour traquer Oussama ben Laden (Gusterson 2016:12) et au fil des attaques, l'Afghanistan gagne le sinistre titre du pays le plus bombardé par des drones au monde (Ross et al. 2014: 3). Ces opérations sont administrées par l'armée américaine qui possède également des troupes au sol, même si les frappes de drones n'ont eu de cesse d'augmenter alors que, simultanément, le nombre de soldats sur le terrain diminuait.

$\mathrm{Si}$, dans le cadre d'une guerre déclarée, le drone vient a priori épauler l'intervention militaire globale, l'usage de la violence dronique est différent dans les pays comme le Yémen, la Somalie et le Pakistan (en particulier dans les régions dites tribales) avec lesquels les États-Unis ne sont pas formellement en guerre, et où le déploiement de troupes terrestres est minimal. De manière significative, la guerre des drones est dès lors menée par la CIA ou le JSOC, le Joint Special Operations Command, plutôt que par l'armée (de l'air) américaine traditionnelle. Dans ce contexte, le recours aux drones représente une forme d'intervention (para)militaire qui prend pour cibles des espaces civils et les individus qui s'y trouvent. Il s'agit d'une stratégie basée sur l'asymétrie et assumée comme telle, selon laquelle la personne désignée comme terroriste est ciblée sans pouvoir répondre à l'attaque, comme cela serait possible sur le champ de bataille traditionnel (Gusterson 2016: 14-15).

Hugh Gusterson, auteur d'un ouvrage de synthèse paru en 2016, identifie quatre raisons principales qui expliquent l'attrait des drones pour l'armée américaine dans le contexte de la guerre " contre le terrorisme». Premièrement, ils représentent l'outil le plus efficace pour effectuer une surveillance généralisée, continue et planétaire. Équipés de caméras, de capteurs infrarouges et de chaleur, et d'autres instruments de mesure, ils enregistrent un flux ininterrompu de données qui sont ensuite analysées dans les centres de renseignements. Deuxièmement, la guerre des drones permet d'éviter les blessés et les morts du côté des soldats américains, ce qui représente un avantage politique important par rapport à la guerre traditionnelle qui implique la mise en danger du personnel militaire national. Troisièmement, les drones restent une option comparativement bon marché et se défendent plus facilement, dans un 
budget militaire, quel'avion de chasse, par exemple. Finalement, un drone promet une attaque d'une précision maximale (Gusterson 2016:21-23). En effet, commel'expliquent également d'autres auteur·e.s, pour les défenseurs de la guerre contemporaine, le drone favoriserait non seulement un conflit "chirurgical, sensible et scrupuleux» (Gregory 2011a: 188) mais aussi une armée plus "petite et maigre» (Shaw 2013: 537). C'est pourquoi ces armes représentent l'option privilégiée de la stratégie antiterroriste du gouvernement américain au moins depuis 2010 (Shaw 2013 : 539).

L'emploi des drones par les États-Unis, concomitant au nombre d’assassinats ciblés, 6 a explosé sous la présidence de Barack Obama. À la fin de son mandat, la presse a rapporté que les 542 frappes autorisées par le président avaient tué plus de 3700 personnes, dont au moins 324 civil.e.s (Zenko 2017). Les chiffres du Bureau of Investigative Jounalism sont sensiblement plus élevés. Pour la même période (2009-2017), il compte en effet « au moins" 430 frappes confirmées rien que pour l'Afghanistan, avec un nombre de morts variant de 2512 à 4026 personnes, dont 969 civil·e.s, parmi lesquels 200 enfants ${ }^{6}$. Ces chiffres sont évidemment éloquents car extrêmement importants. Leur imprécision est également significative. Il est en effet impossible de connaître le nombre exact de victimes et, en l'absence de procédure judiciaire, d’opérer une distinction claire entre les « combattants» et les « civil·e.s» (Shaw 2013:543).

\section{Assemblage, matrice, dispositif : penser la complexité du drone}

La multiplicité des approches mobilisées dans la littérature académique pour comprendre le drone traduit la complexité de l'engin lui-même. Selon Katharine Hall, «la réponse à la question "qu'est-ce qu'un drone” est difficile à cerner, car le drone dépasse toujours le cadre de la question, aujourd'hui comme par le passé. » (Hall Kindervater 2015 : 17) De manière similaire, pour Caren Kaplan et Lisa Parks, le drone touche à des questions de recherche plurielles allant « de la numérisation à la souveraineté, de la surveillance à la géopolitique, du travail à l'affect » (Parks \& Kaplan 2017b : 5). Une analyse critique des drones requiert dès lors de penser l'hybridité de ces objets. Il s'agit d'admettre leur complexité plutôt que de la voiler d'un discours trop simpliste sur leur supposée «nouveauté révolutionnaire».

Dans la littérature anglo-saxonne, plusieurs auteur·e·s se réfèrent à la notion de drone assemblage ("assemblage dronique») pour souligner l'importance de penser le

\footnotetext{
6 Voir les statistiques sur: https://www.thebureauinvestigates.com/projects/drone-war/charts?show casualties $=1 \&$ show_injuries $=1 \&$ show_strikes $=1$ \&location $=$ pakistan $\&$ from $=2004-1-1 \&$ to $=2017-1-1$, consulté le 13 novembre 2019.
} 
drone comme un objet non pas unique et homogène, mais constitué d'éléments hétérogènes et multiformes (Wilcox 2016; Crampton 2016). Empruntée à Gilles Deleuze et Félix Guattari, la notion d'assemblage permet d'accentuer les dimensions à la fois "organiques, mécaniques et sociales de la réalité» (Bousquet 2018: 16). Pour le géographe Derek Grégory, il est par ailleurs essentiel de comprendre le drone comme participant à une nouvelle "matrice de la violence militaire et paramilitaire" qui codétermine ses usages et fonctions (Gregory 2014: 130, je souligne). Dans ce sens, le drone n'est qu'un ensemble, certes central, d'un contexte politique et idéologique plus large qu'il s'agit d'étudier dans son étendue.

Dans le cadre de mes propres recherches en histoire des médias et du cinéma, c'est la notion de "dispositif» qui m'est particulièrement utile. Au milieu des années 1970, Michel Foucault propose une définition qui décrit le dispositif comme un ensemble qui englobe aussi bien des discours que des matérialités, des règles que les institutions qui les portent. Pensé notamment en relation avec l'analyse du Panoptique, le dispositif foucaldien est

"un ensemble résolument hétérogène, comportant des discours, des institutions, des aménagements architecturaux, des décisions réglementaires, des lois, des mesures administratives, des énoncés scientifiques, des propositions philosophiques, morales, philanthropiques, bref: du dit et du non-dit. " (Foucault 1994: 299)

Dans leur discussion du dispositif foucaldien, les historien·ne·s du cinéma François Albera et Maria Tortajada soulignent une distinction importante entre le dispositif en tant qu'«ensemble résolument hétérogène», qu’ils nomment le «dispositifépistémè», et un dispositif matériel et circonscrit tel que le Panoptique. Selon Albera et Tortajada, le second constitue une manifestation emblématique du premier, sans toutefois se confondre avec lui (Albera \& Tortajada 2011: 30-31). Influente pour l'analyse historique d'objets médiatiques, cette double utilisation de la notion permet tout d'abord d'appréhender des dispositifs audiovisuels concrets; dans cette perspective, le « cinéma » ne se limite pas au film mais englobe le spectateur (son corps, son « appareil» psychique), des éléments techniques et architecturaux (machines de projection, salles de cinéma), des institutions et des discours (Albera \& Tortajada 2011). Le dispositif cinéma peut ensuite être aussi envisagé dans sa dimension épistémologique en tant qu'il co-construit les discours, les représentations et les politiques d'un moment historique donné. Le travail de Mireille Berton, par exemple, adopte cette démarche en étudiant les circulations et les échanges de concepts et d'imaginaires entre le 
cinéma des premiers temps et les sciences du psychisme au tournant du $\mathrm{XX}^{\mathrm{e}}$ siècle (Berton 2015). Le cinéma n'est dès lors pas uniquement compris au sein de sa propre histoire, mais comme faisant partie de la culture visuelle, médiatique et scientifique plus globale qu'il contribue à façonner.

Si j'insiste sur cette notion et son utilisation par les historien·ne.s du cinéma dont les travaux ne sont a priori pas directement liés à ceux qui m’intéressent ici -, c'est qu'elle permet d'envisager le drone à la fois comme un objet concret dont les différentes composantes peuvent être décrites, mais également comme un schème - ou

8 une matrice, pour reprendre le terme de Gregory - des politiques militaires contemporaines. Le drone est à la fois une arme qui rend possible certaines stratégies militaires, mais également l'objet paradigmatique de la violence étatique actuelle. Il est à la fois une technologie au service d'un pouvoir donné, et la matérialisation du pouvoir lui-même. Dans le cadre de cette introduction, la notion m’offre en outre un schéma heuristique qui permet de structurer la discussion de la littérature scientifique selon quatre dimensions interdépendantes, à savoir les dimensions matérielle, géographique, médiatique et "corporelle». Ces différentes facettes du dispositif dronique sont traitées séparément pour des raisons pratiques. En réalité, elles s'enchevêtrent pour former ce qu'on peut appeler avec Achille Mbembe la «nécropolitique» du drone, en tant que gestion de "qui pourra vivre et qui devra mourir" (Mbembe 2006: 29; pour une discussion de la notion de Mbembe en lien avec la guerre des drones actuelle, voir Allison 2015 et Wilcox 2016). Le dispositif dronique contemporain est un dispositif nécropolitique, et le décomposer en ses parties interdépendantes montre comment le pouvoir fonctionne à des niveaux à la fois matériels, géographiques, symboliques et politiques.

\section{Les infrastructures droniques}

Alors que le drone est perçu dans l'imaginaire collectif comme un avion " sans pilote», et plus généralement comme une technologie "sans homme» (unmanned), les hommes et femmes nécessaires au fonctionnement du drone armé sont en réalité nombreux. Hugh Gusterson parle en effet de 170 personnes, dont un peu moins de la moitié est requise pour le simple lancement de l'engin (Gusterson 2016:21). Pour le géographe Derek Gregory, ce chiffre est légèrement plus élevé puisqu'il faudrait selon lui 192 personnes, dont 133 travaillant en dehors de la zone de guerre et n'étant donc jamais en danger immédiat (Gregory 2014: 131). Aux ressources humaines s'ajoutent les milliers de kilomètres de câbles en fibre optique, les tonnes de métal et de plastique transformés en satellites, en capteurs et en autres composants, les innombrables bâtiments et 
containers confinés dans les bases militaires, etc. Lisa Parks rappelle en outre la nécessité des ressources naturelles telles que « la surface terrestre, l'air, les fréquences radioélectriques, l'orbite», mobilisées pour faire fonctionner le drone (Parks 2017 : 137).

Cette importante infrastructure matérielle et humaine produit un océan de données virtuelles qui sont à leur tour gérées par l'infrastructure lourde du web. La collecte ininterrompue de données, rendue possible par la surveillance par drones, nourrit les centres de renseignement d'une masse considérable d'informations. Elles y sont traitées par des algorithmes qui dictent alors la loi de vie et de mort. En effet, les frappes de drones reposent essentiellement sur l'analyse computationnelle de données numériques rassemblées dans une disposition matrix. Cette base de données est développée depuis 2010; son objectif est de recenser les terroristes présumés (Weber 2016). Elle produit et distribue les informations des frappes de drones qui s'organisent selon deux principes.

Il y a tout d'abord les "frappes de personnalités" - les personality strikes. Elles visent une personne identifiée en amont et dont les activités terroristes sont supposément prouvées. Selon Grégory Delaplace, ces frappes sont «le plus publicisées " par les autorités américaines, car leur succès matérialise l'idée d'une guerre chirurgicale (Delaplace 2017 : 95). Cependant, la plupart des frappes de drone s'organisent selon un autre principe, celui de la "frappe de signature» ou la signature strike. Celle-ci ne vise pas un individu connu et désigné en amont comme "terroriste», puisque les cibles sont désignées en fonction d'un certain comportement et d'un certain "mode de vie» (pattern of life) (Chamayou 2013: 69 sq.). Cette forme d'assassinat repose en majeure partie sur le calcul algorithmique et non pas sur une expertise humaine: l'analyse des images de surveillance, des images infrarouges, et d'autres données collectées dans la disposition matrix débouche sur l'identification d'attitudes et d'actions supposément suspectes. Or, cette tâche est quasi exclusivement déléguée à la machine (Chamayou 2013; Weber 2016). Pour le géographe Ian Shaw, l'infrastructure technologique planétaire est ainsi à disposition d'un « empire prédateur " qui décide du droit de vie et de mort sur la base d'un pouvoir qui s'affirme à travers « la computation, l'encodage et lélimination de la vie en "temps réel" " (Shaw 2013: 540). La frappe de signature est l'expression inverse d'une guerre "chirurgicale" dans la mesure où elle ne vise pas une personne identifiée en amont qu'il s'agirait d'exterminer avec la plus grande précision, mais des habitudes, des comportements, ou des actions repérés par des systèmes technologiques qui transforment les informations en cibles potentielles. 
L’infrastructure numérique et ses composants - les capteurs de chaleur, les caméras, les bases de données, les satellites, les câbles, les antennes, etc. - représentent finalement le versant matériel d'une nouvelle forme de gouvernance et de guerre qui ne se limite pas à l'analyse d'une situation existante mais qui prétend pouvoir désigner des risques futurs (Weber 2016; Miller 2017). Lintelligence artificielle, le machine learning et les autres possibilités du numérique rendent possible cette nouvelle forme de logistique guerrière où la frappe meurtrière n'est pas réalisée en réponse à une agression, mais intervient de manière préemptive, voire préventive. Comme l'écrit Andrea Miller,

"la guerre par drones est une manifestation de la temporalité anticipatoire qui caractérise les pratiques actuelles de guerre et du policing, où les analyses $d u$ pattern of life et la convergence des données aboutissent à des actions préventives contre les menaces potentielles futures. " (Miller 2017: 116)

Pour Lisa Parks, le dispositif dronique crée ainsi une nouvelle catégorie de population constamment visée par une attaque possible car habitant dans des régions où il y aurait potentiellement des terroristes. Privée des droits fondamentaux, cette "classe ciblée» est également une classe racisée (Parks dans ce numéro). De façon similaire, Miller souligne que ces désignations anticipées de personnes constituant une potentielle future menace répondent à des logiques de racisation des cibles. Le dispositif dronique est raciste puisque la "guerre contre le terrorisme» cible des "corps identifiés en tant que musulmans ainsi que des corps non-musulmans perçus comme musulmans ou arabes" (Miller 2017: 155). Ces corps, dans la logique de la frappe préemptive, représentent, toujours déjà en amont, le potentiel danger.

\section{Spatialités du drone}

Reposant sur un vaste réseau de technologies et de constructions, l'infrastructure dronique s'étend tout autour du globe (Turse 2011; Shaw 2013: 549). La chaîne de commandement est dispersée dans de nombreux centres de renseignement et des bases militaires et repose en outre sur un réseau mondial des télécommunications. La base militaire américaine de Ramstein, en Allemagne, est un exemple emblématique des contraintes géographiques d'une guerre «à distance». Cette dernière nécessite en effet de nombreux appuis locaux à une proximité relative des cibles7. Si un drone $M Q-1$ Predator peut voler 24 heures et parcourir $1250 \mathrm{~km}$ sans toucher terre, il n'est toutefois pas encore possible de mener une guerre globale uniquement depuis les États-Unis.

\footnotetext{
7 Voir le collectif Stopp Air Base Ramstein pour davantage d'informations sur la base américaine: https:// www.ramstein-kampagne.eu/, consulté le 13 novembre 2019.
} 
Récemment, le gouvernement Trump a reconnu être actuellement en guerre contre sept pays (Liautaud 2018). Malgré cette prise de position, les chiffres des conflits impliquant les États-Unis sont contestés et largement tributaires de la manière de définir la nature d'un conflit. Ainsi, selon une carte récente publiée dans le Smithsonian Magazine, les États-Unis seraient engagés dans des activités antiterroristes dans 80 pays, soit dans $40 \%$ des pays du monde ${ }^{8}$. La géographie «multiple et composite» (Gregory 2014:129) du dispositif dronique ne se réduit pas dès lors à un espace guerrier clairement défini. En effet, une des caractéristiques les plus discutées de la guerre des drones est qu'elle peut potentiellement avoir lieu partout. En tant que "everywhere war» (Gregory 2011b), elle abolit le champ de bataille traditionnel et défait les frontières entre des zones de guerre et des zones de paix (Neocleous 2013; Gusterson 2016; Kaplan 2017; voir Sourna Loumtouang dans ce numéro). La licence to kill (Alston 2010) que s'auto-octroient les États-Unis instaure un état de guerre permanent à l'échelle globale, qui «est défini par des batailles asymétriques et paramilitaires dans les régions périphériques de la planète" (Shaw 2013: 537).

Si la guerre des drones est désormais globalisée, elle est aussi individualisée dans la mesure où les stratégies d'assassinat ciblé ne visent pas une armée, et donc une institution collective, mais des corps désignés individuellement - même si, bien évidemment, les "dommages collatéraux» sont toujours élevés et que la guerre par drones cible in fine un "environnement social tout entier» (McSorley 2019: 80). À la fois globalisée et individualisée, cette guerre est aussi centralisée (Shaw 2013: 537). Dans le travail d'investigation publié sous le titre de Drone Papers, Jeremy Scahill et l'équipe de The Intercept détaillent sa place au sein de l'appareil gouvernemental américain. Ils décrivent avec précision le fonctionnement de la dénommée kill chain, ce processus bureaucratique de prise de décision qui rend la nécropolitique américaine possible (Scahill 2015).

Cette multiplicité des spatialités mises en jeu par le dispositif dronique se traduit par le déploiement différencié du pouvoir dans l'espace: le drone permet d'élargir la distance entre le homeland et la zone d'attaque, il transforme le monde en cible potentielle et modèle les zones d'interventions depuis les airs. Ce dernier aspect est une facette centrale du dispositif dronique car il détermine les formes guerrières contemporaines. Comme le résume Grégoire Chamayou : «Il s'agit moins d’occuper un territoire que de le contrôler par le haut en s'assurant la maîtrise des airs.» (Chamayou 2013: 81) De manière similaire, Parks souligne l’importance du «champ vertical» pour comprendre

8 Voir https://www.smithsonianmag.com/history/map-shows-places-world-where-us-military-operates-180970997|, consulté le 13 novembre 2019. 
comment le dispositif intervient sur «la vie sur terre» depuis les airs (voir Parks dans ce numéro). La "verticalisation du pouvoir " se manifeste de manière particulièrement prégnante dans la kill box, un espace désigné à des fins d'attaque aérienne, pensé en trois dimensions. Cette «boîte létale» dessinée sur les écrans des pilotes permet, lorsqu’on l'ouvre, une intervention létale dans un territoire restreint, mais toujours adaptable aux besoins des militaires. Elle est, selon les mots de Chamayou, une "zone autonome de tuerie temporaire» (Chamayou 2013:83, italiques dans le texte). Selon le philosophe, cette violence extrêmement mobile, administrée par les airs et combinée à un arsenal de nouvelles technologies, transforme les géographies guerrières en "terrain de chasse». La guerre des drones n'oppose plus deux ennemis en un face-à-face horizontal sur un champ de bataille, mais confère à l'un l'œil du chasseur qui surveille, verticalement, sa proie (Chamayou 2013).

L'espace létal du drone est aussi un espace légal car la redéfinition continuelle des zones de combats est accompagnée par l'adaptation permanente des lois américaines. Passant outre les législations internationales qui régulent les conflits entre Étatsnations et qui visent notamment à faire respecter les normes humanitaires, la guerre des drones menée par l'armée américaine se fonde sur une state lawfare intense: la loi est en effet utilisée comme arme de guerre par le gouvernement (Hajjar 2017: 6o sq.; Blakeley 2018; voir aussi Brunet dans ce numéro). L’incessante redéfinition des cadres juridiques, censés initialement endiguer les violences en temps de conflit, devient une tactique guerrière. Ici, elle est moins soutenue par une infrastructure algorithmique que par un réseau de juristes et d'avocats influents. Pour ne donner qu'un exemple de cette lawfare américaine: la désignation des "terroristes» comme "unlawful combatants", soit des combattants ennemis illégaux. Cette appellation fait entrer les premiers dans une catégorie qui n'est ni celle des combattants, ni celle des civils, et dont aucune loi ne se préoccupe. Inexistants devant la loi, les unlawful combattants ne sont donc pas des êtres protégés par elle, ou, pour le dire autrement, les institutions censées respecter la loi se donnent le droit d'exemption de cette loi (Hajjar 2017 : 64). La violence extrajudiciaire d'État devient dès lors une réalité bien meurtrière, comme le montre de manière emblématique le cas d'Anwar al-Awlaki, membre d'Al-Qaïda de nationalité américaine, qui a été assassiné par un drone en septembre 2011, un mois avant qu'un autre drone ne tue son fils, Abdulrahman al-Awlaki, âgé de 16 ans et également citoyen américain.

\section{Images et visions droniques}

Les infrastructures et les technologies numériques constitutives des drones sont intrinsèquement liées aux géographies qu'elles déploient. Brouillant les frontières entre 
zones de guerre et zones " civiles », la géographie dronique relève également de la jurisprudence qui légitime certaines redéfinitions de l'espace par le drone. Ces aspects à la fois matériels, politiques et juridiques sont centraux; ils ne couvrent toutefois pas la question du régime de visibilité co-construit par le dispositif.

Ce régime s’inscrit dans l'histoire longue des assassinats ciblés en tant que forme actuelle de la violence coloniale, et plus particulièrement dans l'histoire du bombardement aérien (Adey et al. 2013; Hippler 2014). Une caractéristique fondamentale du régime scopique de la vue aérienne, que parfait le drone contemporain, est d'offrir une hypervisibilité qui s'apparente à un regard omniscient, englobant et ubiquitaire (Kaplan 2006; Wilcox 2016; Maurer 2016). À cet égard, le nom donné au système vidéo qui équipe les drones est parlant : Gorgon Stare - le regard de la Gorgone (Gregory 2011a : 193). Cette hypervisiblité est rendue possible grâce à l'automatisation de la vision qui, comme le rappelle Claus Gunti dans sa discussion des images droniques en tant qu’images "automatisées", est facilitée par l'émergence des technologies numériques à la fin des années 1990. Enrichies par des métadonnées et destinées à être analysées par des machines, ces images créent une représentation du monde «sans le concours d'une quelconque intervention humaine» (Gunti 2014: 66). Pour Francisco Klauser et Silvana Pedrozo, les "politiques de visibilité» du drone doivent justement être comprises en tant qu'enchevêtrement "de l'humain et du non-humain" (Klauser \& Pedrozo 2015 : 287). De manière analogue, dans son histoire de la perception militaire, Antoine Bousquet montre que la convergence de la vision et de l'annihilation est basée sur une pluralité de technologies et de pratiques qui font converger l'œil et la machine dans la quête de l'arme parfaite (Bousquet 2018). La «logistique de la perception " (Virilio 1984) telle qu'elle est administrée par l’armée met au pas les corps et les machines.

Le regard dronique de la Gorgone en tant que régime d'hypervisibilité n'est ainsi pas séparable de la violence létale qui définit le dispositif. Dans son étude sur l'histoire des drones, Katharine Hall explique que le drone associe les deux pratiques principales des armées modernes: le renseignement, la surveillance et la reconnaissance (ISR en anglais), d'une part, et la mort à distance, de l'autre. En faisant converger le regard panoptique et une machine à tuer, la "surveillance létale» du drone constitue le perfectionnement le plus abouti de l'articulation de l'œil et de la bombe (Hall Kindervater 2016). Suivant la même logique, Caren Kaplan suggère que l'image dronique est «basée sur un régime scopique militarisé beaucoup plus étendu et complet que toute forme antérieure de surveillance et de reconnaissance» (Kaplan 2017 : 169). 
Finalement, parce qu'il conjugue la perception à l'acte de tuer, le régime scopique du drone peut être utilement appréhendé avec la notion d'image "opérationnelle» que propose Harun Farocki dans son analyse de la guerre du Golfe. Pour le cinéaste, l'image opérationnelle est une image qui fait et qui agit le monde plus qu'il ne le représente (Farocki 2004). Ainsi, les écrans qu’observent les opérateurs depuis leurs containers ne montrent pas simplement des représentations d'un paysage enregistré par un drone et ne se cantonnent pas à diffuser des images augmentées par métadonnées, calculées et lues par la machine. Ce que les écrans montrent se traduit par une action concrète de la part de la team qui aboutit, dans le «meilleur » des cas, à l'assassinat à

14 distance. L'image opérationnelle du drone est essentielle à la nécropolitique du dispositif dans son ensemble.

\section{Les corps mis en jeux}

La dimension sensorielle et affective de la guerre des drones constitue le dernier aspect du dispositif dronique. Elle s'ajoute à la dimension visuelle et ne doit pas être éclipsée par l'idée d'une guerre ultra-technologisée - voire robotisée. En effet, la guerre des drones n’est ni "aseptisée» ni "propre»; elle met en jeu des corps des deux côtés de l'écran. Comme le soulignent en particulier des chercheuses mobilisant des approches de genre (gender), l'expérience du drone est une expérience incarnée qui s'inscrit dans et transforme les corps qu'elle touche (Holmqvist 2013; Wilcox 2016; Parks dans ce numéro).

Il est particulièrement important de redonner existence aux corps pour deux raisons au moins. Premièrement, cette démarche permet de se défaire de la notion centrale mais problématique de l'engin "sans homme», le Unmanned Aerial Vehicle, qui alimente l'idée d'une guerre à "zéro morts». Remettre au centre de l'attention les corps faits et défaits par les drones est une manière de nommer les victimes assassinées et de réintroduire les pertes de vies humaines dans le discours public ainsi que la douleur et les blessures vécues par des communautés entières. À ce titre, le travail des ONG qui se sont attelées à compter les morts est particulièrement précieux (Rogers 2010; Cavallaro et al. 2012). Il permet de contredire les rapports officiels du gouvernement américain en mettant en avant le chiffre extrêmement élevé de personnes assassinées par drone, et surtout le ratio entre les supposés "terroristes" tués et les "civil·e·s", "dommages collatéraux» de cette guerre. Donnant une voix aux survivant.e.s d'attaques de drones, les rapports laissent aussi entrevoir les traumatismes psychiques, émotionnels et collectifs des communautés touchées. Ainsi, réincarner la guerre des drones permet d'en faire la critique et de déconstruire l'idée d’une guerre « chirurgicale». 
Deuxièmement, l'attention portée aux expériences corporelles et individuelles des victimes permet de mieux comprendre comment les drones modifient durablement les communautés qu'ils menacent. Kevin McSorley insiste sur la dimension sonore et sensorielle du dispositif dronique, que l'on retrouve également dans les rapports des ONG réalisés auprès de victimes. Le bourdonnement de l'engin, les silences après une attaque et l'attente de la suivante, mais aussi l'impossibilité d'organiser des réunions en groupe sont autant de signes d'un monde affectif modifié (McSorley 2019). Mobilisant la notion de "médiation verticale", Lisa Parks signale les effets du dispositif dronique qui se déploie à la fois dans les airs et sur le sol, effets qui «réécrivent et reforment la vie sur terre de la manière la plus matérielle" (Parks 2018: 147; pour la notion de "médiation verticale», voir son article dans ce numéro). Affectant les populations ciblées - que Parks appelle les classes ciblées - à travers la mort et la destruction, le drone est "autant une technologie d'inscription qu'une technologie de détection ou de représentation" (Parks 2018: 147). Pour les victimes des attaques et leurs communautés, les drones inscrivent littéralement la nécropolitique dans les paysages, les villages et les corps.

À cet égard, on peut mentionner une stratégie particulièrement cruelle qui vise à déstabiliser le tissu communautaire des populations touchées : la stratégie du double tap, soit de la frappe double. Cette tactique consiste à attaquer deux fois de suite afin de frapper les personnes qui auraient pu venir au secours des premières victimes (Gusterson 2016: 96; Shaw 2013: 543-544). Lattaque de funérailles de victimes de drones représente une méthode similaire (Delaplace 2017: 113). Bien loin de l'image d'une guerre technologique et donc supposée sophistiquée, ce qui transparaît ici est plutôt la chasse à l'homme décrite par Chamayou, dont l'objectif premier est « de prévenir l'éclosion de menaces émergentes par l'élimination précoce de leurs agents potentiels" (Chamayou 2013: 54), et ceci indépendamment du fait que les "agents potentiels" puissent être des enfants, des femmes, des terroristes ou des civil.e.s.

De l'autre côté de l'écran, qui sépare et simultanément réunit le chasseur et sa proie, on trouve les pilotes de drones. Les chercheuses en études genre et queer discutent la façon dont la guerre par drone - qui extirpe le soldat du champ de bataille - déstabilise les définitions traditionnelles de la masculinité militaire, de même que les notions de virilité, de courage ou de prise de risque (Daggett 2015; Bayard de Volo 2016; Sandeau dans ce numéro). Or, le dispositif dronique touche également à l'intégrité physique des pilotes, comme le mettent en lumière de nombreux récits qui soulignent la difficulté de retrouver une vie "normale» après le service militaire. 
Sans mettre sur le même plan des formes de souffrance qui ne sont pas comparables, il est important de signaler les effets de ce que Peter Asaro nomme «l'assassinat bureaucratique" sur la santé mentale des soldats (Asaro 2017; voir aussi Férey dans ce numéro). Il s'agit non pas de justifier leurs actes ni de minimiser leurs responsabilités, mais de montrer que, si la guerre des drones est bien une guerre à «zéro morts" du côté américain, elle laisse néanmoins des traces non négligeables dans le homeland.

\section{Penser le dispositif du drone}

16 Les articles réunis dans ce numéro abordent plusieurs des questions qu'esquisse cette introduction et apportent des éclairages sur les dimensions géo/politiques, matérielles, psychologiques, juridiques et pratiques du drone. Ils contribuent à nourrir la discussion actuelle sur les drones à travers un regard pluridisciplinaire mais aussi des approches originales. Le numéro est en effet en dialogue constant avec les "Drone Studies " anglosaxonnes dont il reprend les propositions, mais ajoute également des perspectives plus rarement explorées dans la littérature existante.

Le dossier a bénéficié d'une journée de workshop organisée à l'Université de Lausanne en mars 2019. Elle a permis une discussion collective des articles et donné lieu à un partage stimulant de réflexions autour d'un objet commun. Par ailleurs, les échanges continus avec les auteur·e-s pendant la phase de finalisation des textes a contribué à renforcer la cohérence de l'ensemble.

Le dossier s'organise autour de deux parties principales. Il réunit tout d'abord les contributions qui analysent le drone armé, en particulier américain. Les articles appréhendent la guerre des drones comme un phénomène à la fois militaire et juridique, qui se déroule non seulement sur des zones d'interventions lointaines mais qui a aussi un impact dans le homeland. Le numéro rassemble ensuite les textes qui proposent plusieurs déplacements par rapport à la focalisation sur la guerre des drones américaine actuelle. Ces contributions étudient en effet d'autres usages, d'autres territoires, d'autres périodes ou abordent encore l'objet via la question des représentations culturelles. Ces textes se penchent ainsi sur les drones civils ou policiers, sur les enjeux du drone dans le continent africain ou durant la Deuxième Guerre ou encore sur l'imaginaire des drones dans la culture populaire. Ces déplacements permettent de nourrir une réflexion sur des usages de drones qui dépassent le champ de bataille contemporain et d'affiner l'analyse critique de l'objet. 
Le dossier s'ouvre par la traduction d'un article de Lisa Parks paru en 2016 dans la revue Feminist Studies. Peu connue dans l'espace francophone, la chercheuse américaine se dédie depuis plusieurs années déjà à l'analyse critique des drones. La traduction de son texte permet de donner accès à ses travaux dans l'espace francophone et d'introduire sa réflexion sur les "champs verticaux" et leurs enjeux de pouvoir. Pour Parks, en effet, «les drones ne flottent pas simplement au-dessus de la surface du globe; au contraire, ils réécrivent et restructurent la vie sur terre de la manière la plus matérielle qui soit." Une conséquence directe de cette restructuration est l'émergence de ce qu'elle nomme la "classe ciblée», à savoir une population qui est continuellement la cible potentielle des attaques par drones «simplement parce qu'elle vit et se déplace dans des zones où il est possible que des terroristes présumés opèrent».

Le texte de Parks est traduit par Marie Sandoz qui signe également le deuxième article, une introduction aux travaux de la chercheuse américaine. Cette présentation parcourt les recherches de Parks depuis sa thèse de doctorat jusqu'à ses dernières publications. Elle s'attache à mettre en évidence les continuités des réflexions de Parks, qui abordent les questions des infrastructures médiatiques et de l'espace aérien, conceptualisé à travers la notion de «champ vertical». En lien avec le texte traduit, Sandoz signale notamment la place de la création artistique dans la démarche de Parks, pour qui l'art constitue «un lieu où les visions dominantes des technologies peuvent être dénoncées et subverties aussi bien qu'un creuset où se forment des conceptions émancipatrices". De cette manière, l'historienne souligne le double intérêt des travaux de Parks: s’il s'agit de fournir une analyse critique et engagée des drones, la chercheuse réfléchit également aux possibilités d'action et aux potentiels espaces de résistance face au pouvoir dominant.

Si l'article de Lisa Parks mobilise la notion de "classe ciblée» pour penser l'asymétrie structurelle qui fonde les guerres des drones, Amélie Férey inverse la perspective et se penche sur les effets de la guerre des drones sur les pilotes. Elle analyse en particulier les états de stress post-traumatique (ESPT) qui touchent ceux-ci. Alors que l'armée américaine utilise la présence de l'ESPT chez les pilotes de drone comme preuve du fait que la guerre à distance n'équivaut pas à un «jeu vidéo ", mais implique le personnel au même titre que les soldats sur le terrain, Férey met en garde contre ces discours qui font un lien trop rapide entre le mal-être du soldat et son empathie présumée. S’appuyant notamment sur la théorie freudienne des névroses de guerre, elle montre qu'une source centrale de l'ESPT n'est pas à chercher dans la relation au 
champ de bataille mais plutôt dans la proximité entre le champ des opérations (souvent un container dans une base militaire sur sol américain) et l'espace domestique. Ainsi, pour Férey, l'ESPT reflète moins « une prise de conscience de l'acte de donner la mort et une éventuelle culpabilité» que les conditions professionnelles du soldat qui se voient chamboulées par la guerre à distance.

L'article de Quentin Brunet s'inscrit également dans le cadre d'une analyse des guerres des drones principalement américaines, mais cette fois pour interroger une notion centrale du discours de légitimation des frappes, celle du "terroriste». Prenant comme point de départ la citation de Noam Chomsky, qui affirme que l'État américain est le plus grand terroriste international, Brunet étudie les appropriations de cette notion par différents acteurs de la guerre des drones. L'auteur avance l'argument suivant: «tant pour les Américains que pour les groupes qu'ils combattent, l'enjeu consiste à s'éloigner de l'appellation de terroristes afin de légitimer leur combat et de le représenter comme une guerre juste." Le concept de guerre juste, dont la définition s'appuie sur des notions comme celle du "terrorisme», est lui-même l'enjeu d'une guerre juridique que Brunet analyse dans le sillage de Lisa Hajjar et d'autres en tant que state lawfare. Sa contribution met ainsi en lumière les dimensions juridiques et discursives de la guerre des drones. Il montre que celle-ci ne se déroule pas uniquement dans des zones ciblées (relativement) lointaines, mais également dans des bureaux d'avocats et dans les pages de la presse occidentale.

Larticle d'Anne-Katrin Weber opère quant à lui un premier déplacement de la focale en proposant une étude historique de l'émergence des drones durant la Deuxième Guerre mondiale. Pour cela, l'auteure s'intéresse à la conceptualisation de dispositifs télévisuels qui apparaissent dans les années 1930 et dont les potentialités militaires seront mobilisées durant le conflit pour ensuite être réadaptées à la période de paix. La télévision militaire devient alors "industrielle». Cette inscription de l'analyse des drones dans l'histoire des médias permet de souligner les circulations des technologies, des pratiques et des concepts entre les domaines militaires et civils. Larticle nourrit de la sorte la réflexion actuelle sur l'omniprésence des drones (civils) dans nos sociétés en y apportant un éclairage historique.

De son côté, la contribution d'Erick Sourna Loumtouang analyse l'usage militaire et civil des drones sur le continent africain, et en particulier dans le bassin du lac Tchad. Le texte s’intéresse à une région que l'auteur décrit comme «la dernière frontière des drones». Pour Sourna Loumtouang, si les drones promettent de résoudre 
des problèmes infrastructurels, notamment dans le domaine de la santé, ils sont également l'expression d'une "nouvelle modalité d'impérialisme sur fond technologique», matérialisé par "la multiplication des bases de drones sur le continent" dont la construction se justifie par la "guerre contre le terrorisme", et en particulier par la présence de Boko Haram. L’analyse de la prolifération des aéronefs sans équipage sur le territoire africain permet à l'auteur de souligner la complexité politique des drones, "ballotés entre applications civiles, applications militaires et logiques impérialistes». La contribution met en outre en évidence les intérêts économiques et stratégiques des États-Unis, de la France et de la Chine, notamment, dans le développement de la technologie sur le continent.

Le texte de Silvana Pedrozo et Francisco Klauser est une contribution originale de par sa démarche empirique qualitative fondée sur des entretiens et des observations de terrain auprès de la Police neuchâteloise. L'étude des deux géographes vise à retracer les processus d'intégration des technologies de drones au sein de l'institution policière. Grâce à leur enquête, Pedrozo et Klauser montrent les mécanismes informels et formels qui dirigent l'acquisition de la nouvelle technologie, et mettent en lumière la part importante « de facteurs individuels et personnels - tels que les motivations et connaissances techniques et pratiques de l'individu qui introduit l'objet dans son nouveau milieu - dans la découverte institutionnelle du drone». Ainsi, au-delà de l'analyse minutieuse d'un terrain donné, Pedrozo et Klauser soulèvent la question de l'utilisation des drones dans des institutions non militaires et pointent la nécessité d'un débat politique et sociétal qui devrait accompagner la dissémination de ces dispositifs.

Hélène Jeannin prend comme objet d'étude le projet Maven de Google et la contestation qu'il a déclenchée en 2018 aux États-Unis chez salarié·e·s de l'entreprise. À travers l'analyse de ce débat sociétal, la sociologue éclaire le rôle de l'intelligence artificielle (IA) dans le développement actuel du drone et son impact sur le renforcement de l'imbrication des secteurs privé, militaire et gouvernemental américains. En effet, pour Jeannin, «l'écosystème du drone est tissé d'un réseau d’interdépendances entre acteurs privés et publics, que ce soit sous l'angle de la R\&D, de l'opérationnel, ou des financements." La contestation à l'interne de Google face à un programme de recherche sponsorisé par le Department of Defense pose dès lors le cadre d'une réflexion ouverte sur les enjeux éthiques et sociétaux de l'usage des drones, et plus largement sur l'utilisation de l'IA militaire. 
Le numéro se clôt sur l'article de Jules Sandeau qui s'intéresse non pas directement à la guerre des drones elle-même mais à sa représentation dans la culture populaire. À partir de films hollywoodiens récents, l'analyse de Sandeau se demande comment la culture populaire traite l'arrivée des machines sans équipage, qui questionne de façon multiple la masculinité guerrière traditionnelle. Alors que le cinéma hollywoodien célèbre l'avènement de nouvelles technologies comme le drone, il est également animé par " une volonté de sauvegarder l’idéal dominant de masculinité militaire». En adoptant une perspective d'études genre, Sandeau analyse ainsi la manière dont les films négocient, "dans le domaine de l’imaginaire», "des tensions idéologiques » découlant 20 de l'usage accru des drones par l’armée américaine. À l’instar des deux articles précédents, sa contribution met finalement l'accent sur la présence des drones dans nos propres espaces de vie en étudiant leur prolifération sur nos petits et grands écrans.

En définitive, les articles de ce volume mettent en évidence les dimensions multiples du dispositif dronique à travers un dialogue interdisciplinaire. Ce dialogue semble d'autant plus urgent et nécessaire que de nombreuses questions politiques, éthiques et philosophiques sont d'une actualité brûlante et pourraient bénéficier de l'intervention informée de chercheuses et chercheurs adoptant les perspectives des sciences sociales et humaines pour analyser le drone en tant qu'objet concret ainsi que les formations de pouvoir auxquelles il donne lieu.

\section{Remerciements}

J'aimerais remercier chaleureusement les personnes qui ont contribué à la réalisation de ce numéro: Antonin Wiser, rédacteur en chef d'a contrario, pour son soutien tout au long du processus d'élaboration et de publication; les membres du comité d'a contrario pour les relectures et la discussion avec les auteur.e.s; Marie Sandoz et Claire-Lise Debluë pour avoir soigneusement relu et commenté mes propres textes; Mireille Berton pour les discussions "dispositif», encore et toujours; Sacha Auderset pour la mise en page et sa patience; et bien sûr les auteur.e.s pour leurs contributions.

Le workshop organisé en amont de la publication a bénéficié du soutien financier du Centre d'études cinématographiques de l'Université de Lausanne (CEC) et du Fonds national suisse de la recherche scientifique (FNS). 


\section{Références}

Adey Peter, Whitehead Mark \& Williams Allison (dir.) (2013), From Above: War Violence, and Verticality, Oxford, Oxford University Press.

Albera François \& Tortajada Maria (2011), "Le dispositif n'existe pas!», in Cinédispositifs: spectacles, cinéma, télévision, littérature, F. Albera et M. Tortajada (dir.), Lausanne, L'Âge d'Homme, pp. 13-38.

Allison Jamie (2015), "The Necropolitics of Drones ", International Political Sociology, vol. $9, \mathrm{n}^{\circ}$ 2, pp. 113-127.

Alston Phillip (2010), Study on Targeted Killings. Report of the Special Rapporteur on extrajudicial, summary or arbitrary executions, United Nations. URL: https://www2. ohchr.org/english/bodies/hrcouncil/docs/14session/A.HRC.14.24.Add6.pdf, consulté le $1^{\mathrm{er}}$ octobre 2019.

AsARo Peter (2017), "The Labor of Surveillance and Bureaucratized Killing. New Subjectivites of Military Drone Operators ", in Life in the Age of Drone Warfare, L. Parks et C. Kaplan (dir.), Durham/Londres, Duke University Press, pp. 282-314.

BAYARD DE Volo Lorraine (2016), «Unmanned? Gender Recalibrations and the Rise of Drone Warfare ", Politics \& Gender, vol. 12, $\mathrm{n}^{\circ}$ 1, pp. 50-77.

BERTON Mireille (2015), Le corps nerveux des spectateurs. Cinéma et sciences du psychisme autour de 1900, Lausanne, L'Âge d'Homme.

BLA KELEY Ruth (2018), “Drones, State Terrorism and International Law », Critical Studies on Terrorism, vol. 11, $\mathrm{n}^{\circ}$ 2, pp. 321-341.

BOUSQUET Antoine (2018), The Eye of War. Military Perception from the Telescope to the Drone, Minneapolis/Londres, University of Minnesota Press.

Cavallaro James, Sonnenberg Stephan \& Knuckey Sarah (2012), Living under Drones: Death, Injury, and Trauma to Civilians from US Drone Practices in Pakistan, Stanford, International Human Rights and Conflict Resolution Clinic, Stanford Law School; New York, NYU School of Law, Global Justice Clinic.

Снам аyou Grégoire (2013), Théorie du drone, Paris, La Fabrique.

CRAM PTON Jeremy (2016), "Assemblage of the Vertical: Commercial Drones and Algorithmic Life", Geographica Helvetica, n 71, pp. 137-146.

DAGgetT Cara (2015), « Drone Disorientations: How “Unmanned” Weapons Queer the Experience of Killing in War», International Feminist Journal of Politics, vol. 17, $\mathrm{n}^{\circ}$ 3, pp. 361-379.

Dela Place Grégory (2017), "Comment pensent les drones. La détection et l’identification de cibles invisibles ", L’Homme, vol. 2, nº 222, pp. 91-118.

FARocki Harun (2004), «Phantom Images », Public. Art, Culture, Ideas, n 29, pp. 13-22. 
Fouc Ault Michel (1994), "Le Jeu de Michel Foucault », in Dits et écrits. 1954-1988, Paris, Gallimard, pp. 298-332.

GREGORY Derek (2011a), «From a View to a Kill: Drones and Late Modern War », Theory, Culture \& Society, vol. 28, nos 7-8, pp. 188-215.

GREGORY Derek (2011b), "The Everywhere War ", The Geographical Journal, vol. 177, nº 3 , pp. 238-250.

GREGORY Derek (2014), "Géographies du drone», Décadrages. Cinéma, à travers champs, $\mathrm{n}^{\text {os }}$ 26-27, pp. 129-150.

Gunti Claus (2014), "L'image automatisée entre drones et appropriations", Décadrages. Cinéma, à travers champs, $\mathrm{n}^{\text {os }}$ 26-27, pp. 66-93.

Gusterson Hugh (2016), Drone: Remote Control Warfare, Cambridge, MIT Press.

HaJjA Risa (2017), «Lawfare and Armed Conflicts. A Comparative Analysis of Israeli and U.S. Targeted Killing Policies and Legal Challenges against Them ", in Life in the Age of Drone Warfare, L. Parks et C. Kaplan (dir.), Durham/Londres, Duke University Press, pp. 59-88.

Hall Kindervater Katharine (2015), Lethal Surveillance: Drones and the Geo-History of Modern War, manuscrit de thèse, University of Minnesota. URL: https://conservancy. umn.edu/handle/11299/175214, consulté le 18 novembre 2019.

Hall Kindervater Katharine (2016), "The Emergence of Lethal Surveillance: Watching and Killing in the History of Drone Technology", Security Dialogue $47, \mathrm{n}^{\circ} 3$, pp. 223-238.

HiPPLER Thomas (2014), Le gouvernement du ciel: Histoire globale des bombardements dériens, Paris, Les Prairies ordinaires.

HolmQvist Caroline (2013), "Undoing War: War Ontologies and the Materiality of Drone Warfare", Millennium: Journal of International Studies, vol. 41, $\mathrm{n}^{\circ}$ 3, pp. 535-552.

KAPLAN Caren (2006), "Mobility and War: The Cosmic View of US "Air Power" ", Environment and Planning A, vol. 38, $\mathrm{n}^{\circ}$ 2, pp. 395-407.

KAPLAN Caren (2017), "Drone-O-Rama: Troubling the Temporal and Spatial Logics of Distance Warfare ", in Life in the Age of Drone Warfare, L. Parks et C. Kaplan (dir.), Durham/Londres, Duke University Press, pp. 161-177.

Klauser Francisco \& Pedrozo Silvana (2015), "Power and Space in the Drone Age: a Literature Review and Politico-Geographical Research Agenda ", Geographica Helvetica, $\mathrm{n}^{\circ} 70$, pp. 285-293.

Liautaud Alexa (2018), "White House Acknowledges the U.S. Is at War in Seven Countries", Vice, 16 mars 2018. URL: https://www.vice.com/en_us/article/a3ywd5/whitehouse-acknowledges-the-us-is-at-war-in-seven-countries, consultéle 13 novembre 2019.

MAURER Kathrin (2016), "Visual Power : The Scopic Regime of Military Drone Operations ", Media, War \& Conflict, vol. 10, $\mathrm{n}^{\circ}$ 2, pp. 141-151. 
Mвемве Achille (2006), “Nécropolitique», Raisons politiques, vol. 21, nº 1, pp. 29-6o.

MCSORLEY Kevin (2019), " Predatory War, Drones and Torture: Remapping the Body in Pain ", Body \& Society, vol. 25, $\mathrm{n}^{\circ}$ 3, pp. 73-99.

Miller Andrea (2017), "(Im)Material Terror: Incitement to Violence Discourse as Racializing Technology in the War on Terror", in Life in the Age of Drone Warfare, L. Parks et C. Kaplan (dir.), Durham/Londres, Duke University Press, pp. 112-133.

Neocleous Mark (2013), "Air Power as Police Power ", Environment and Planning D: Society and Space, vol. 31, pp. 578-593.

PAR Ks Lisa (2017), "Vertical Mediation and the U.S. Drone War in the Horn of Africa", in Life in the Age of Drone Warfare, L. Parks et C. Kaplan (dir.), Durham/Londres, Duke University Press, pp. 134-157.

PARKs Lisa (2018), Rethinking Media Coverage. Vertical Mediation and the War on Terror, New York/Londres, Routledge.

PARKS Lisa \& KA Plan Caren (dir.) (2017a), Life in the Age of Drone Warfare, Durham/ Londres, Duke University Press.

PARKS Lisa \& KAPLAN Caren (2017b), "Introduction", in Life in the Age of Drone Warfare, Durham/Londres, Duke University Press, pp. 1-21

Rogers Christopher \& Center FOR Civilians in Conflict (2010), Civilian in Armed Conflicts. Civilian Harm and Conflict in Northwest Pakistan. URL: https://civiliansinconflict.org/wp-content/uploads/2017/og/Pakistan_Report_2010_2013.pdf, consulté le 10 octobre 2019.

Ross Alice K., SERle Jack \& Will Tom (2014), Tracking Drone Strikes in Afghanistan: A Scoping Study, The Bureau of Investigative Journalism. URL : https:// v1.thebureauinvestigates.com/wp-content/uploads/2014/07/TBIJ-Afghanistan-Report. pdf, consulté le 18 novembre 2019.

SCA HILl Jeremy \& The Intercept (2015), "The Drone Papers », The Intercept. URL: https:/ theintercept.com/drone-papers, consulté le 28 novembre 2019. Les textes réunis sur ce site ont été traduits en français et sont disponibles sous forme de livre: Jeremy Scahill et l'équipe de The Intercept (2017), La machine à tuer: La guerre des drones, Montréal, Lux Éditeur.

SH AW Ian G. R. (2013), "Predator Empire: The Geopolitics of US Drone Warfare», Geopolitics, vol. $18, \mathrm{n}^{\circ} 3$, pp. 536-559.

TURSE Nick (2011), "The Pentagon's Planet of Bases», TomDispatch.com. URL: http:/ www.tomdispatch.com/blog/175338/nick_turse_the_pentagon\%27s_planet_of_bases; consulté le 18 novembre 2019.

Virilio Paul (1984), Guerre et cinéma I. Logistique de la perception. Paris, Cahiers du Cinéma/Éditions de l'Étoile.

Weber Jutta (2016), « Keep Adding. On Kill Lists, Drone Warfare and the Politics of Databases ", Environment and Planning D : Society and Space, vol 34, $\mathrm{n}^{\circ}$ 1, pp. 107-125. 
\{́́ditorial

Wilcox Lauren (2016), "Embodying Algorithmic War: Gender, Race, and the Posthuman in Drone Warfare", Security Dialogue, vol. $48, \mathrm{n}^{\circ}$ 1, pp. 11-28.

Zenko Micah (2017), “Obama's Final Drone Strike Data», Council on Foreign Relations, 20 janvier 2017. URL: https://www.cfr.org/blog/obamas-final-drone-strike-data, consulté le 13 novembre 2019.

Zubeldia Océane (2912), Histoire des drones, Paris, Perrin. 